was the question which most concerned the participants at the conference.

The chemists seemed to be interested chiefly in the effects of extreme-pressure additives on the load-carrying capacities of oils. For instance, Dr E. S. Forbes (BP Research Centre, Sunbury-onThames) demonstrated that the loadcarrying ability of the alkylammonium dialkyl phosphates and dithiophosphates depended markedly on their structure. Dr E. V. Zaretsky (NASA Lewis Research Centre, Cleveland) described a fourteenfold increase in life obtained by adding a substituted organic phosphate to a actual ball bearing being run with synthesized hydrocarbon oil under a low oxygen environment and at extreme pressures. This was a vivid illustration of the improvement obtainable by forming chemically a protective film between surfaces loaded beyond that point at which normal lubrication breaks down.

The presence of oxide films on the surfaces of worn metals was the subject of several contributions. Oxide films clearly have a protective role in that they prevent intermetallic contact. The oxidation of the metal, however, must also lead to an increased wear, if the oxide film cannot remain intact during sliding. Drs T. F. J. Quinn (University of Aston in Birmingham) and N. Tenwick (Queen Mary College, London) dealt with particular oxidational wear models. Both these workers underlined the need for a thorough examination of the kinetics of oxidation during wear, because the results of static oxidation experiments are apparently not relevant to the conditions occurring at the wearing interface.

The extremely reactive nature of clean metal surfaces (such as one has when the protective oxide film breaks up under cyclic mechanical or thermal stresses caused by sliding) was emphasized by Dr D. W. Morecroft (Shell Research, Thornton, Chester). He described some high vacuum experiments in which typical hydrocarbons were shown to react much more quickly with an oxide-free surface of iron than they would have done on the normal oxide-covered surface.

\section{PALAEONTOLOGY}

\section{New Techniques}

from a Correspondent

THE annual Christmas meeting of the Palaeontological Association held in Belfast from January 5-7 firmly established that the renewed vitality of the palaeontological sciences owed much to the application of new techniques from peripheral disciplines. The small size of the meeting alone acted as an effective means of furthering communication between active workers involved in interdisciplinary approaches from Britain, Ireland, Europe and North America.
The sixteen contributions covered a wide range of topics outlining the consequences of, and future developments arising from, isotope studies, electron microscopy, organic geochemistry, biochemistry, biological cultures, films of behaviour and numerical studies. The programme effectively divided into two spheres of interest: the functional and environmental, and the numerical. The key trend in developmental studies arising from all these approaches is towards a detailed understanding of the environments in which the fossils lived, secreted their skeletons and were buried, and the subsequent changes which affected them and their matrices. By contrast the chief advances in numerical studies are largely directed towards data storage, retrieval and evolutionary prediction.

Two encouraging trends emerged from a lively discussion from the floor. First, after years of separatist specialization, palaeontologists are finding that trends in skeletal development, as revealed by combined mineralogical, biochemical and physiological approaches, are yielding a uniform end-product irrespective of phylum. Thus a common language and understanding of processes is developing as was shown by a comparison of investigations on the "shell" structures of brachiopods (Professor A. Williams, Queen's University, Belfast), bryozoa (Dr R. Tavener-Smith, Queen's University, Belfast) and molluscs (Dr J. Kennedy, Oxford). These studies use a combination of ultrastructural and light microscopic techniques in the comparison of the role of Recent soft tissues in calcification and diagenesis with well preserved fossil material which elucidate phyletic trends and classificatory problems.

Second, after several years of quantitative bewilderment, it seems that the application of computers to palaeontolo-

\title{
Fluctuations in Seismic Activity
}

IN next Monday's Nature Physical Science, G. F. Davies and J. N. Brune return to a problem which first attracted the famous seismologist Hogo Benioff just twenty years ago. Using an improved technique, they have apparently succeeded in detecting significant fluctuations in seismic activity over the seventy years or so since accurate measurements of earthquake magnitude were begun.

Benioff's original analysis involved calculation of the cumulative strain released by the world's large earthquakes between 1904 and 1951 from the sum of their energies. The result showed that during the 46 year period there had been five active phases separated by quiescent periods and that the cycles thus produced had become successively shorter. This was, and remains, a surprising conclusion. The deep seated global processes which account for seismic activity presumably have characteristic time scales running into millions of years. How, then, could they exhibit periodicities of a few tens of years or even less?

It was partly because of the unsatisfactory nature of Benioff's conclusion, and subsequent criticism of the way it was arrived at, that Davies and Brune have carried out a new analysis using a different technique. Their method involves the calculation of slip rates in fault zones by summing the seismic moments of the large (Richter magnitude greater than 7.0), shallow (depth less than $60 \mathrm{~km}$ ) earthquakes within them. In essence, this is very little different from the Benioff method. Where it scores, however, is that the relationship between seismic moment and magnitude is much simpler than that between seismic energy and magnitude, and so fewer assumptions are required. Thus, for example, whereas Benioff was strongly criticized for assuming that earthquake volume is independent of magnitude, Davies and Brune have neatly sidestepped the problem by effectively eliminating it.

Not surprisingly, the new analysis fails to confirm the existence of five cycles. What it does indicate, though, is a fivefold decrease in seismic activity since 1897 Indeed, the global data suggest three distinct periods of activity marked by well defined discontinuities. So although there seem to be no periodicities as such, Davies and Brune, like Benioff, are also faced with the problem of reconciling vastly different time scales, once accepting that the decrease with time is statistically significant. This they do by invoking mechanical coupling through lithospheric plates-an option which was not, of course, open to Benioff. In other words, they seek to explain the fluctuations in seismic activity by suggesting that the largest earthquakes are not independent.

The second result obtained by Davies and Brune clearly gives them greater satisfaction. Most of the slip rates they have calculated for particular seismic regions are agreed in general with the rates calculated independently by Le Pichon (J. Geophys. Res., 73, 3661; 1968) from plate tectonic theory. Obviously this is very satisfactory indeed, though in view of the many simplifying assumptions Davies and Brune still found necessary, it is also quite surprising. Even more surprising is the fact that the slip rate for the past twenty years is almost exactly equal to the average rate over the past ten million years obtained by Oliver and Isaaks (Canad. J. Earth Sci., 5, 985; 1968). 